\title{
Characterization of genome-wide segmental duplications reveals a common genomic feature of association with immunity among domestic animals
}

Xiaotian Feng ${ }^{1+}$, Jicai Jiang ${ }^{1+}$, Abinash Padhi ${ }^{2}$, Chao Ning ${ }^{1}$, Jinluan $\mathrm{Fu}^{1}$, Aiguo Wang ${ }^{1}$, Raphael Mrode ${ }^{3}$ and Jian-Feng Liu ${ }^{1 *}$

\begin{abstract}
Background: Segmental duplications (SDs) commonly exist in plant and animal genomes, playing crucial roles in genomic rearrangement, gene innovation and the formation of copy number variants. However, they have received little attention in most livestock species.

Results: Aiming at characterizing SDs across the genomes of diverse livestock species, we mapped genome-wide SDs of horse, rabbit, goat, sheep and chicken, and also enhanced the existing SD maps of cattle and pig genomes based on the most updated genome assemblies. We adopted two different detection strategies, whole genome analysis comparison and whole genome shotgun sequence detection, to pursue more convincing findings. Accordingly we identified SDs for each species with the length of from $21.7 \mathrm{Mb}$ to $164.1 \mathrm{Mb}$, and 807 to 4,560 genes were harboured within the SD regions across different species. More interestingly, many of these SD-related genes were involved in the process of immunity and response to external stimuli. We also found the existence of 59 common genes within SD regions in all studied species except goat. These common genes mainly consisted of both UDP glucuronosyltransferase and Interferon alpha families, implying the connection between SDs and the evolution of these gene families.
\end{abstract}

Conclusions: Our findings provide insights into livestock genome evolution and offer rich genomic sources for livestock genomic research.

Keywords: Domestic animals, Segmental duplications, Copy number, Duplicated genes

\section{Background}

Repetitive DNA sequences are ubiquitous and these duplicated sequences occupy almost half of the human genome [1]. One type of DNA sequences among various repetitive sequences, with high sequence similarity $(\geq 90 \%)$ and longer than $1 \mathrm{~kb}$, is called segmental duplication (SD). SDs tend to cluster within subtelomeric and pericentromeric regions, and the high similarity of SDs

\footnotetext{
* Correspondence: liujf@cau.edu.cn

${ }^{\dagger}$ Equal contributors

${ }^{1}$ National Engineering Laboratory for Animal Breeding, Key Laboratory of Animal Genetics, Breeding and Reproduction, Ministry of Agriculture, College of Animal Science and Technology, China Agricultural University, Beijing 100193, China

Full list of author information is available at the end of the article
}

can lead to genomic rearrangement and recombination [2-5]. SDs are associated with non-allelic homologous recombination (NAHR) which may facilitate the formation of copy number variations (CNVs) [6-8]. SDs have been considered to play an important role in gene innovation, where genes embedded show a significant enrichment of biological functions in immunity, growth and responses to external stimuli [1, 9-12]. Recently, functional studies have unravelled that genetic diseases like Williams-Beuren syndrome and infertility are associated with genomic rearrangement caused by SDs on chromosomes 7 and $\mathrm{Y}$, respectively, in the human genome $[13,14]$. 
With the progress of sequencing projects moving forward, it is possible to explore the distribution, features and potential roles of duplicated sequences in genome evolution. Since the pioneer studies on SDs in human genome, several studies have been performed aiming at identification and characterisation of genome-wide SDs among other mammalian species such as mouse [9], rat [10], chimpanzee [11] and dog [12].

Although SDs are considered as one of the most important structural features in mammalian genomes, they have received little attention in most livestock species. So far, SDs have been merely systematically investigated in the genomes of bovine and swine $[15,16]$. Liu et al. [16] reported a SD map of the bovine genome based on the version of bovine reference genome Btau 4.0.. Recently, we have constructed a SD map of the porcine genome based on the reference genome of Sscrofa10.2 [17], but the unmapped scaffolds have been largely ignored for SD detection therein.

For most of other livestock species, i.e., horse, sheep, goat, rabbit and chicken, etc., seldom studies have been performed in-depth for SD characterization. Aiming at enhancing the understanding of the roles of SDs in genomic innovation and functional characterization of SDs across different species, we conducted global identification and comparison of SDs across seven livestock species in the current study. We applied two commonly used methods, i.e., whole-genome assembly comparison (WGAC) and whole-genome shotgun sequence detection (WSSD) $[3,18]$ to explore genome-wide SDs in the genome of each species investigated. Our objectives herein lie in two aspects. Firstly, we present comprehensive SD profiles and comparison across the genomes of various livestock species, which will be beneficial to relevant studies on structural and functional genomics as well as evolutionary genetics related to SD regions; Secondly, we characterized and annotated SD regions across different species' genomes to provide global insights into genomic structural features, further exploring potential functional genes and common mechanisms corresponding to $\mathrm{SD}$ regions.

\section{Methods}

\section{Genome resources of domestic animals}

All genomic data for SD analyses are from publicly-accessible databases. Genome assemblies for pig (Sscrofa10.2) [19], cattle (UMD3.1) [20], horse (EquCab2.0) [21], rabbit (OryCun2.0) [22], sheep (Oar_v3.1) [23] and chicken (Gallus_gallus-4.0) [24] were downloaded from Ensembl databases (ftp://ftp.ensembl.org/pub/), and those of cattle (Btau 4.6.1) [25] and goat (CHIR_1.0) [26] were downloaded from the NCBI FTP site (ftp://ftp.ncbi.nlm. nih.gov/genomes/). Meanwhile, we downloaded next generation sequencing (NGS) data of the individual of the reference genome for each species, i.e., NGS data of porcine from the DDBJ FTP site (ftp://ftp.ddbj.nig.ac.jp/ ddbj_database/dra/fastq/ERA009/ERA009086/), ovine and caprine from the NCBI FTP site (ftp://ftp-trace.ncbi.nlm. nih.gov/sra). Whole genome shotgun sequencing (WGS) sequence data of cattle, horse, rabbit and chicken were also downloaded from the NCBI FTP site (ftp://ftp-tra ce.ncbi.nlm.nih.gov/sra), which were then spliced to 36bp to simulate NGS data for WSSD analyses [27]. The resources of gene families were downloaded from HGNC database (HUGO Gene Nomenclature Committee, http:// www.genenames.org/genefamilies/a-z).

\section{Segmental duplication detection}

We used two different approaches to detect SDs in the genomes of seven domestic species, i.e., WGAC and WSSD methods. All the details to implement the two approaches were illustrated in our previous study [17].

After finishing both WGAC and WSSD analyses for the reference genome, to further remove artifactual duplications, we filtered the WGAC alignments of $\geq 94 \%$ identity using the WSSD dataset. Following previous studies $[9,10,12,16,18]$, the final SD database consisted of the combined results from the WGAC approach with identity $<94 \%$ and the rest part filtered using the results of the WSSD approach (all custom Perl scripts are available at https://github.com/jiang18/sd_analysis). Finally, we constructed SD maps of domestic animals using the program Parasight v7.6 (http://eichlerlab.gs.washington.edu/jeff/ parasight/index.html).

\section{Analyses of gene content within SD regions}

We retrieved gene contents within SD regions based on genome annotation files downloaded from NCBI (e.g., ftp://ftp.ncbi.nih.gov/genomes/Sus_scrofa/mapview/seq_g ene.md.gz). Bioconductor (http://www.bioconductor.org/) was used to perform Gene Ontology (GO) analyses. Kyoto Encyclopedia of Genes and Genomes (KEGG) pathway analyses were conducted with DAVID (http://david.abc c.ncifcrf.gov/). Since only a limited number of genes in the livestock genomes have been annotated, we firstly converted the gene IDs of investigated livestock species to orthologous human Ensembl gene IDs by BioMart (http://www.biomart.org/), then carried out the GO and KEGG pathway analyses. We also analyzed orthologous protein-coding genes within SD regions among domestic animals based on OrthoDB release 7 (http://cegg.unige.ch/ orthodb7). The phylogenetic trees were drawn using Clustal X (http://www.clustal.org/clustal2/) and Tree View (http:// taxonomy.zoology.gla.ac.uk/rod/treeview.html).

\section{Association with other genomic landscapes}

To further characterize identified SDs, we performed simulations to probe whether the identified SDs are associated 
with other genomic features, like CNVR, subtelomeric and pericentromeric regions and gene family regions. The simulation analyses were done by our self-developed Perl scripts. To test for association between SDs and CNVRs, we randomly assigned each of identified SD regions a putative position with no overlap with each other in the genome. The number or the length of CNVRs overlapping with SDs was calculated in each simulation, and finally, we created empirical distributions of the hits via 10,000 independent replications. Thus the significance of SD enrichment in CNV regions could be determined by the thresholds based on the empirical distributions. Similarly, associations of SDs with subtelomeric and pericentromeric regions as well as gene family regions were performed based on the same strategy. For the enrichment analyses, we defined approximate lengths of both subtelomeric and pericentromeric regions as $2 \mathrm{Mb}$ based on previous studies of karyotype of each species [16, 28-36]. Considering the differences between avian genome and mammalian genome, subtelomeric and pericentromeric regions of several chromosomes in chicken genome were shortened to $300 \mathrm{~kb}$.

\section{Results}

\section{Identification of segmental duplications}

We identified segmental duplications among domestic animals based on two different approaches. Wholegenome assembly comparison (WGAC) is a BLASTbased approach to identify alignments with length $\geq 1 \mathrm{~kb}$ and identity $\geq 90 \%$ [3], while whole-genome shotgun sequence detection (WSSD) can find SD regions by calculating mapping read depth [18, 37]. After removing "artifactual duplications", we identified the SD regions among domestic animals by combining the filtered results of WGAC approach and the results of WSSD approach.

For WGAC analyses, the initial results were significantly different among the seven species investigated, ranging from 54,933 pairwise alignments (goat) to 902,537 pairwise alignments (pig). After removing highcopy repeats, the number of pairwise alignments for most of the investigated species reduced to $\sim 20,000$ and the rabbit genome had the largest amount of alignments, with 54768 (Table 1). The number of alignments decreased in porcine genome dramatically, which may be due to the filtration of initial alignments of high similarity. According to previous studies, SDs showed a significant enrichment in unassigned scaffolds $[3,12,16]$. Compared with other 6 species, rabbit genome has larger number of unassigned scaffolds $(17.9 \%, 489.7 \mathrm{Mb}$ of $2,737.4 \mathrm{Mb})$, which may account for its larger number of pairwise alignments.

Specifically, we identified 31,148 pairs of alignments in the Btau 4.6 genome assembly for cattle, among which
Table 1 The amount of initial and cleaned pairwise alignments for each of 7 domestic species investigated

\begin{tabular}{lll}
\hline Species & Initial alignments & Cleaned alignments \\
\hline Cattle & 63,978 & 16,402 \\
Pig & 902,537 & 20,000 \\
Horse & 70,532 & 14,841 \\
Rabbit & 338,613 & 54,768 \\
Goat & 54,933 & 14,403 \\
Sheep & 107,362 & 11,719 \\
Chicken & 111,922 & 25,823
\end{tabular}

The initial WGAC pairwise alignments were filtered by custom Perl programs. Duplications with 50 or more copies within the genome or present on 3 or more chromosomes were removed, generating cleaned pairwise alignments

18,872 (60.6\%) involved unmapped scaffolds. In contrast, only 1,019 in 13,946 pairs of alignments involved unmapped alignments in the UMD 3.1 assembly. Btau 4.6 is the sole livestock genome assembly with the $\mathrm{Y}$ chromosome in our study. Surprisingly, 9,954 pairs of alignments (32.0\%) involved the Y chromosome, among which 3793 pairs (38.1\%) were linked to unmapped scaffolds. Since we were more interested in chromosomes than unmapped scaffolds, we focused on UMD 3.1 for further analyses of cattle genome.

The identity distributions of alignments are showed in Fig. 1. The curve of alignments with identity from 9096\% largely keeps constant in most of the investigated species, while varying significantly out of this interval among different domestic species. Accordingly, in the identified interval of $96-100 \%$, the distribution curves of porcine, ovine, caprine and chicken alignments with identity $\geq 94 \%$ need to be filtered with results of WSSD approach to remove "artifactual duplications".

In WSSD analyses, there were 4,994, 924, 1,829, 1,226, 2,028, 1,959 and $948 \mathrm{SD}$ intervals (with length $\geq 10 \mathrm{~kb}$ ) identified for cattle, pig, horse, rabbit, goat, sheep and chicken, respectively (Additional file 1: Table S1.1-7). Average absolute copy numbers of these intervals ranged from 6.7 (rabbit) to 12.0 (pig) and for each species were 9.1, 12.0, 10.1, 6.7, 11.6, 11.3 and 6.9, respectively.

After removing "artifactual duplications", we finally determined the SD contents of seven domestic species. For bovine, porcine, equine, rabbit, caprine, ovine and chicken genome, the SD contents of the genome were $2.6 \%$ (68.2 Mb of 2,670.4 Mb), 2.0\% (57.3 Mb of 2,808.5 $\mathrm{Mb}), 6.6 \%$ (164.1 Mb of 2,474.9 Mb), 5.1\% (139.7 Mb of 2,737.5 Mb), 3.4\% (90.2 Mb of 2,635.8 Mb), 3.3\% (87.0 $\mathrm{Mb}$ of 2,619.0 Mb) and 2.0\% (21.7 Mb of 1,100.5 Mb), respectively (Additional file 2: Table S2, Additional file 3: Table S3.1-7). These contents were similar to other mammalian species studied before, like dog [12] and human [18]. The chicken genome with the smallest reference genome had the lowest content. We conjectured 


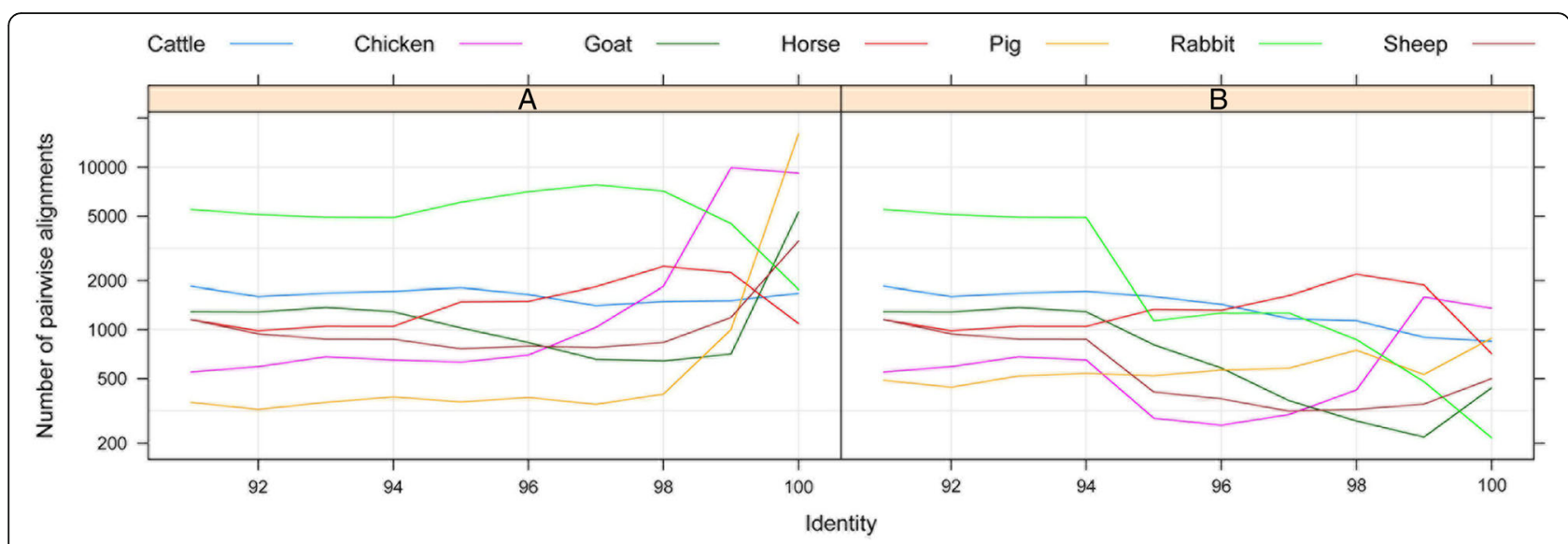

Fig. 1 Identity distribution of pairwise alignments. Panel a shows the identity distribution of pairwise alignments based on the cleaned results of WGAC approach, while panel $\mathbf{b}$ displays the WGAC results filtered by WSSD approach. Each color represents one species

that SD content depends on the scale of reference genome and the unmapped scaffolds. Finally, we constructed SD maps of seven domestic species (Additional file 4: Figure S1.1-7).

We specifically investigated the proportion of WGAC detected long SDs ( $>10 \mathrm{~kb},>94 \%$ similarity) verified by the WSSD results (Table 2). A low proportion implied that the genome assembly had a more serious issue in distinguishing SDs.

\section{Distribution of segmental duplications}

SD regions were dispersed across the genome for each of the investigated species. We calculated total length of SDs on each chromosome for seven domestic species (Additional file 4: Figure S2.1-7, Figure S3.1-7).

Interestingly, SD regions for most investigated species (5 out of 7 species, including cattle, pig, horse, goat and sheep) were overlong in the $\mathrm{X}$ chromosome, especially for cattle and goat. Notably, in chicken genome, chromosome 26 had no pairwise alignments detected by WGAC approach, and no duplicated region with length $\geq 10 \mathrm{~kb}$ identified by WSSD approach as well. Due to the poor annotation of chicken genome [38], no
SDs in chromosome W was identified by both two approaches (only 10 short segments were detected in W_Random chromosome).

For bovine, porcine, equine, rabbit and chicken genomes, intrachromosomal duplications were much more than interchromosomal duplications excluding unmapped scaffolds. For porcine, equine and chicken genome, interchromosomal duplications had higher sequence identity than intrachromosomal duplications. Inversely in the caprine and rabbit genomes, the majority of alignments between chromosomes had a low sequence identity of $\leq 94 \%$.

Previous studies revealed that SDs account for high proportion of contents on unmapped scaffolds [1, 9-12, 16, 39]. Except porcine genome, over $10 \%$ of unmapped scaffolds were identified as SD regions and the proportion reaches $40 \%$ for equine genome (44.1 out of 107.9 $\mathrm{MB})$. The enrichment of SDs in unmapped scaffolds in these domestic species was similar to previous studies and the high identity of SDs became a tremendous obstacle encountered when we mapped these segments to reference genome.

Similar to human, mouse and dog genomes $[1,9,12]$, SDs were enriched in subtelomeric and pericentromeric

Table 2 Copy numbers of genes in SD regions for domestic species

\begin{tabular}{|c|c|c|c|c|}
\hline Species & Genome assembly & Length of WGAC hits $>10 \mathrm{~kb}$ & $\begin{array}{l}\text { Length of WGAC hits }>10 \mathrm{~kb} \\
\text { filtered by WSSD }\end{array}$ & Proportion of filtered WGAC hits \\
\hline Cattle & Btau 4.6 (excluding Y) & $69,558,932$ & $51,891,155$ & 0.746 \\
\hline Cattle & UMD 3.1 & $19,740,894$ & $18,397,219$ & 0.932 \\
\hline Pig & Sscrofa 10.2 & $23,482,697$ & $20,311,340$ & 0.865 \\
\hline Horse & EquCab 2.0 & $59,554,690$ & $56,165,607$ & 0.943 \\
\hline Rabbit & OryCun 2.0 & $24,688,548$ & $16,130,349$ & 0.653 \\
\hline Goat & CHIR 1.0 & $2,093,238$ & $1,946,680$ & 0.930 \\
\hline Sheep & Oar_v3.1 & $1,044,992$ & 888,894 & 0.851 \\
\hline Chicken & Ggallus 4.0 & $3,020,167$ & $2,268,769$ & 0.751 \\
\hline
\end{tabular}


regions among seven domestic species. Because of the imprecise determination of telomeric and centromeric regions of domestic species, we considered approximate subtelomeric and pericentromeric regions based on previous studies [28-31, 34, 36, 40]. SDs of these seven domestic species showed significant enrichment in pericentromeric regions, i.e., 5.5-fold $(P<0.0001)$ for bovine genome, 4.8 -fold $(P<0.0001)$ for porcine genome, 8.7-fold $(P<0.0001)$ for equine genome, 1.8 -fold $(P<$ $0.0001)$ for rabbit genome, 9.3-fold $(P<0.0001)$ for caprine genome, 3.8-fold $(P<0.0001)$ for ovine genome and 3.5fold $(P<0.0001)$ for chicken genome. For subtelomeric regions, SDs were enriched with 1.8 -fold $(P<0.0001)$, 16.4fold $(P<0.0001)$, 3.6-fold $(P<0.0001), 2.8$-fold $(P<0.0001)$, 2.7-fold $(P<0.0001), 1.8$-fold $(P<0.0001)$ and 2.3-fold $(P<$ $0.0001)$ for cattle, pig, horse, rabbit, goat, sheep and chicken, respectively. This indicated that the enrichment of SDs in subtelomeric and pericentromeric regions occurred in majority of domestic species.

The repeat properties of SD regions among domestic species were summarized in Additional file 5: Table S4. The content of each repeat category was similar with each other among six mammalian species, while an obviously different feature existed in the chicken genome in contrast to other six mammalian species. Specifically, the DNA elements of SDs in chicken genome was slightly less than mammalian genome, while the average length of SDs in chicken genome was nearly twice longer than that of SDs in mammalian genomes; For long interspersed elements (LINEs) and short interspersed elements (SINEs), both the number and the average length of the avian genome was extremely lower than those of mammalian species.

\section{Gene content of segmental duplications}

Based on the gene information of each species from NCBI, we found 3,734, 3,096, 3,690, 2,924, 2,460, 4,560 and 807 genes in SD regions identified in bovine, porcine, equine, rabbit, caprine, ovine and chicken genomes, respectively. We calculated the copy numbers of those genes. Average copies of genes ranged from 4.8 to 11.9 (11.9 for bovine genome, 7.3 for porcine genome, 5.5 for equine genome, 4.8 for rabbit genome, 4.9 for caprine genome, 5.5 for ovine genome and 6.6 for chicken genome). Half of genes had more than two copies, mainly ranging from 3 to 10 copies (Table 3 ).

To in-depth exploit potential functions of genes within SD regions among various species, we performed Gene Otology (GO) and KEGG pathway enrichment analyses on all genes within SD regions for each species surveyed. Overall, similar to the results of previous studies in human [18], mouse [9], rat [10], chimpanzee [11], dog [12] and silkworm [39], we found that genes in SD regions were largely enriched with functions and process of
Table 3 Proportion of WGAC-detected SDs verified by WSSD

\begin{tabular}{|c|c|c|c|c|c|c|c|}
\hline \multirow[b]{2}{*}{$C N$} & \multicolumn{6}{|c|}{ Species } & \multirow[b]{2}{*}{ Chicken } \\
\hline & Cattle & Pig & Horse & Rabbit & Goat & Sheep & \\
\hline$\leq 1.5$ & 39 & 19 & 29 & 21 & 4 & 6 & 7 \\
\hline $1.5-2.5$ & 644 & 712 & 604 & 694 & 924 & 1146 & 149 \\
\hline $2.5-10.5$ & 1049 & 720 & 1522 & 1000 & 670 & 927 & 463 \\
\hline $10.5-20.5$ & 300 & 66 & 134 & 114 & 34 & 64 & 32 \\
\hline $20.5-30.5$ & 72 & 14 & 29 & 22 & 2 & 5 & 20 \\
\hline $30.5-40.5$ & 26 & 1 & 15 & 5 & 3 & 2 & 3 \\
\hline $40.5-50.5$ & 15 & 2 & 5 & 3 & 1 & 2 & 4 \\
\hline $50.5-60.5$ & 7 & 0 & 4 & 2 & 1 & 0 & 3 \\
\hline $60.5-70.5$ & 6 & 1 & 0 & 3 & 2 & 1 & 1 \\
\hline $70.5-80.5$ & 4 & 1 & 1 & 0 & 3 & 0 & 2 \\
\hline $80.5-90.5$ & 4 & 1 & 1 & 0 & 0 & 0 & 2 \\
\hline $90.5-100.5$ & 1 & 0 & 0 & 0 & 0 & 2 & 2 \\
\hline$\geq 100.5$ & 41 & 13 & 5 & 1 & 3 & 9 & 1 \\
\hline Null & 1527 & 1547 & 1340 & 1059 & 814 & 2396 & 119 \\
\hline Total & 3735 & 3097 & 3689 & 2924 & 2461 & 4560 & 808 \\
\hline Ave.CN & 11.9 & 7.3 & 5.5 & 4.8 & 4.9 & 5.5 & 6.6 \\
\hline
\end{tabular}

immunity, growth and responses to external stimuli for most of these mammalian species.

Specially, for GO terms, we found that genes in SD regions of five species (dog, cattle, pig, horse and sheep) were commonly enriched in xenobiotic metabolic process and response to xenobiotic stimuli (Additional file 6: Table S5.1). For molecular function ontology, genes of most species (8 out of 10 species, except goat and chicken) were enriched in glucuronosyltransferase activity which is related to drug metabolism (Additional file 6: Table S5.2) [41]. Different from mammalian species, genes in SD regions of the chicken genome were mainly enriched in cell projection organization and neuron projection development. This may due to the differences of evolution course between chicken and mammalian species. In pathway enrichment analyses, those significant pathway-enriched genes in most species were mainly associated with detoxification and metabolism process (Additional file 7: Table S6). It is notable that the olfactory transduction pathway contains the largest amount of olfactory receptor genes in bovine, porcine, equine and rabbit genomes. These olfactory receptor proteins have been reported as one of the main duplicated gene families [42-44].

To seek the exact genes commonly embedded in SD regions among different species, we converted IDs of genes of livestock species to human homologous gene IDs for further comparison. We picked out a total number of 304 common genes within SD regions of at least five species (listed in Additional file 8: Table S7). We then investigated whether these 304 common genes were enriched in certain 
pathways and involved in some common biology processes (Table 4). Accordingly, we found that these common genes played a crucial role in the enrichment of immunity and response to external stimuli. Considering the relatively poor gene annotation in caprine genome as well as the specialization of chicken genome, we finally determined 59 genes as mutual genes in SD regions among domestic species including cattle, pig, horse, rabbit and sheep (Fig. 2, Additional file 9: Table S8). These 59 SD-harbored common genes mainly belong to four gene families, i.e., UDP glucuronosyltransferases (UGTs), interferons (IFNs), histones and olfactory receptors (ORs). Intriguingly, both of UGTs and IFN gene families are significantly enriched in SD regions $(P<0.0001)$ across the genomes of all livestock species. The phylogenetic trees of detected genes of UGT2 and IFN- $\alpha$ families within SD regions for 5 mammalian species were showed in Fig. 3. Previous reports have shown that UGTs transfer the glucuronic acid component of UDP-glucuronic acid to a small hydrophobic molecule which is associated with xenobiotic metabolic process in liver [45], and IFNs are the proteins for defencing external viruses which is made and released by host cells [46]. This provides an important evidence on the potential roles of SDs associated with immunity and responses to external stimuli due to the functions of these two gene families being widely present in the SD regions across the genomes of majority of mammalian species.

\section{Association of SDs with gene families}

It has been reported that gene duplication and conversion are important sources of the evolution of gene families, including those with uniform members and those with diverse functions [47]. To explore association between SDs and various gene families, we further investigated potential enrichment of gene families in SD regions. We firstly collected the gene families from human genome HGNC database and mapped them to the corresponding livestock genome investigated according to the orthology between human and each of species. We then tested the enrichment of gene families in the corresponding genome via simulation based on two different criteria, i.e., the length of genes overlapping with SD regions as well as the number of genes involved in SD regions. As shown in Table 5, we found that gene families were enriched in SD regions $(P<0.001)$ in contrast to non-family genes among common domestic species.

\section{Gene orthology within SD regions}

To survey common features of SDs across various livestock species, we sifted out a total number of 89 orthologous genes within SD regions of all livestock species according to the resources of OrthoDB [48] (Additional file 10: Table S9). Surprisingly, we found orthologous genes in SD regions also showed enrichment of immune response, olfactory receptor activity, G-protein coupled

Table 4 KEGG pathway enrichment analysis of common genes in SD regions among domestic species

\begin{tabular}{|c|c|c|c|c|c|}
\hline KEGG Term & Description & Count & $P$-Value & Fold Enrichment & Bonferroni \\
\hline hsa00980 & Metabolism of xenobiotics by cytochrome P450 & 22 & $8.33 \mathrm{E}-20$ & 14.92 & 7.00E-18 \\
\hline hsa00982 & Drug metabolism & 22 & $1.84 \mathrm{E}-19$ & 14.43 & $1.55 \mathrm{E}-17$ \\
\hline hsa00830 & Retinol metabolism & 20 & $4.83 \mathrm{E}-18$ & 15.07 & 4.05E-16 \\
\hline hsa00983 & Drug metabolism & 16 & $2.32 \mathrm{E}-14$ & 15.14 & $1.95 \mathrm{E}-12$ \\
\hline hsa00140 & Steroid hormone biosynthesis & 16 & 7.37E-14 & 14.15 & $6.18 \mathrm{E}-12$ \\
\hline hsa05320 & Autoimmune thyroid disease & 15 & $7.57 \mathrm{E}-12$ & 11.96 & $6.36 \mathrm{E}-10$ \\
\hline hsa04740 & Olfactory transduction & 35 & $7.62 \mathrm{E}-12$ & 3.76 & $6.40 \mathrm{E}-10$ \\
\hline hsa04140 & Regulation of autophagy & 13 & $1.34 \mathrm{E}-11$ & 15.11 & $1.13 \mathrm{E}-09$ \\
\hline hsa04623 & Cytosolic DNA-sensing pathway & 14 & $3.49 \mathrm{E}-10$ & 10.35 & 2.93E-08 \\
\hline hsa04612 & Antigen processing and presentation & 16 & $8.22 \mathrm{E}-10$ & 7.84 & $6.90 \mathrm{E}-08$ \\
\hline hsa04622 & RIG-I-like receptor signaling pathway & 14 & $1.01 \mathrm{E}-08$ & 8.02 & $8.45 \mathrm{E}-07$ \\
\hline hsa00053 & Ascorbate and aldarate metabolism & 8 & $6.88 \mathrm{E}-08$ & 19.14 & $5.78 \mathrm{E}-06$ \\
\hline hsa00040 & Pentose and glucuronate interconversions & 8 & $1.10 \mathrm{E}-07$ & 18.08 & $9.26 \mathrm{E}-06$ \\
\hline hsa04620 & Toll-like receptor signaling pathway & 14 & 7.61E-07 & 5.64 & $6.39 \mathrm{E}-05$ \\
\hline hsa00150 & Androgen and estrogen metabolism & 9 & $2.13 \mathrm{E}-06$ & 9.90 & 1.79E-04 \\
\hline hsa00500 & Starch and sucrose metabolism & 9 & 5.87E-06 & 8.72 & 4.93E-04 \\
\hline hsa00860 & Porphyrin and chlorophyll metabolism & 8 & $1.09 \mathrm{E}-05$ & 9.86 & 0.000916 \\
\hline hsa04650 & Natural killer cell mediated cytotoxicity & 14 & $1.72 \mathrm{E}-05$ & 4.28 & 0.001442 \\
\hline hsa00591 & Linoleic acid metabolism & 7 & $4.52 \mathrm{E}-05$ & 10.17 & 0.003790 \\
\hline hsa04630 & Jak-STAT signaling pathway & 14 & 8.71E-05 & 3.67 & 0.007294 \\
\hline
\end{tabular}




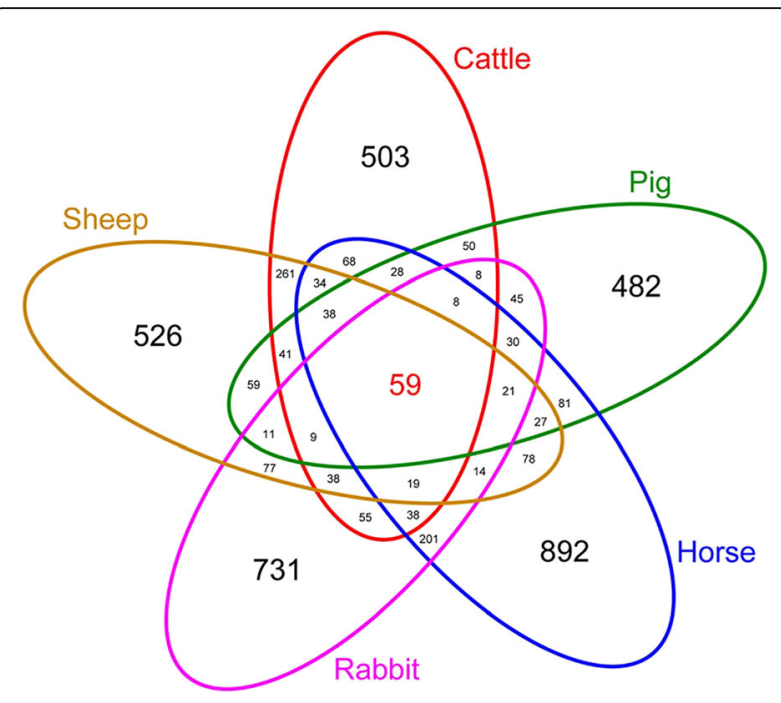

Fig. 2 Common genes among 5 mammalian domestic species. The Venn diagram shows the number of common genes among 5 mammalian domestic species. Each color represents one species

receptor activity and sensory perception of smell. Furthermore, we found that the orthology group EOG6R518B commonly presented among all nine species except pig, which were mainly associated with functions of carboxypeptidase activity and signal transduction.

\section{Discussion}

To our knowledge, this is the first global analysis of segmental duplications among a majority of domestic animals. We identified genome-wide SDs in bovine, porcine, equine, rabbit, caprine, ovine and chicken genomes. The distribution and features of SDs in mammalian domestic species were similar to previous studies in rat and mouse, while SDs in the chicken genome had obviously different characteristics. Fifty-nine common genes were identified in SD regions across five mammalian domestic species and showed significant enrichment in immunity function and responses to external stimuli. Our studies presented valuable resources for further systematic investigation of duplicate blocks, duplicate genes and CNV formation. This will benefit the genome assemblies of domestic species with better understanding of these duplicated sequences on unmapped scaffolds as well. It is notable that the SDs detected were based on the reference genomes released before the beginning time of current study. It should be preferable to employ the latest version of the reference genome to update the SD database herein in our future endeavours.

As we all known, segmental duplications are long DNA sequences (typically defined as being $>1 \mathrm{~kb}$ in length) that have nearly identical sequences (90-100\%) and exist in multiple locations as a result of duplication events. However, there are three possible outcomes when large nearly identical duplicated sequences are encountered during sequence and assembly: (1) The sequences may be recognized as distinct and properly resolved as separate loci, (2) the sequences may be underrepresented due to the presence of virtually identical sequence already in the database, or (3) distinct paralogous loci may be mistakingly assembled into a single sequence contig [4]. Example, In the SD study of human, It had been discussed the likelihood that highly similar (for example, $>98 \%$ identity) apparent intrachromosomal duplications may be erroneous [18, 49]. Meanwhile, It realized that many duplicated regions in current, published genome sequences are in fact errors due to mis-assembly [50]. Therefore, the complete genome were more prior to correct the false segmental duplications caused by genome mis-assembly and detect more accurate segmental duplications.

Chicken is the first sequenced domestic species and is a crucial avian livestock in many countries [51]. However, unmapped scaffolds still took up $4.0 \%$ of the chicken genome. According to our study, over $1 / 10$ (7.2 Mb of $68.6 \mathrm{Mb})$ of these unmapped sequences consisted of segmental duplications. These high-identity sequences are obstacles for genome assembly. The chicken genome showed different SD features from mammalian domestic species. No SDs in chromosome W were identified in our study. This may be due to the limited genetic diversity of chromosome W which is influenced by sex-linked selection [52]. Totally different from mammalian species, genes in SD regions in the chicken genome showed enrichment in cell projection organization and neuron projection development which shared no similar function with those in mammalian species.

In our study, we found that all the investigated mammalian livestock showed enrichment of SDs in subtelomeric and pericentromeric regions. Besides, genes harboured in SD regions were enriched in immunity functions and responses to external stimuli in most of the mammalian animals.

Based on our results, over half of genes in SD regions have multi-copies ranging from 4.8 to 11.9 . We found 11 genes with more than 5 copies among all of our investigated domestic animals as well as in human, mouse and dog genome. Interestingly, most of these multi-copy genes were pseudo genes and were associated with sexrelated functions. In bovine genome, a tandem cluster of pseudo genes on chromosome 17 were found in SD regions, which were associated with testis-specific Yencoded protein. According to previous studies, testis specific protein Y-encoded (TSPY) was a tandem cluster of genes with multi-copies ranged from 50-200 copies in cattle genome [53, 54]. Zinc finger (ZNF) genes were found in all domestic species. This gene family was also reported as tandem gene clusters in mammalian genomes $[55,56]$. In human genome [57], ZNF gene clusters were 

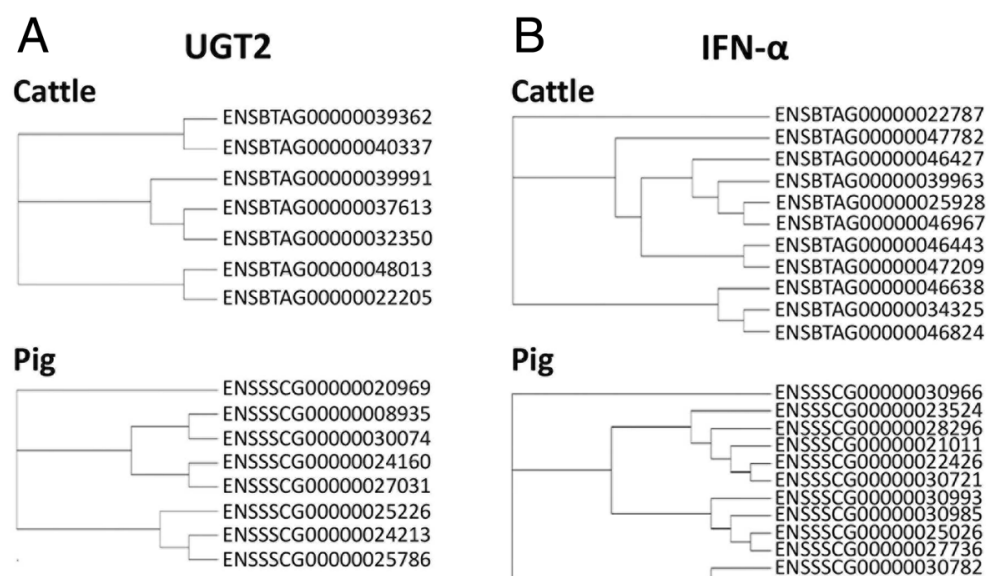

Pig

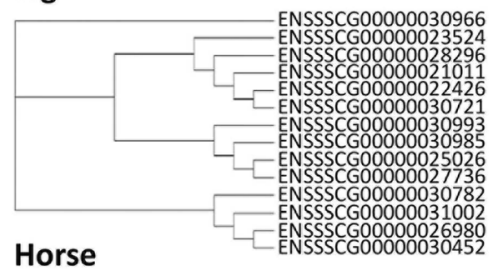

Horse
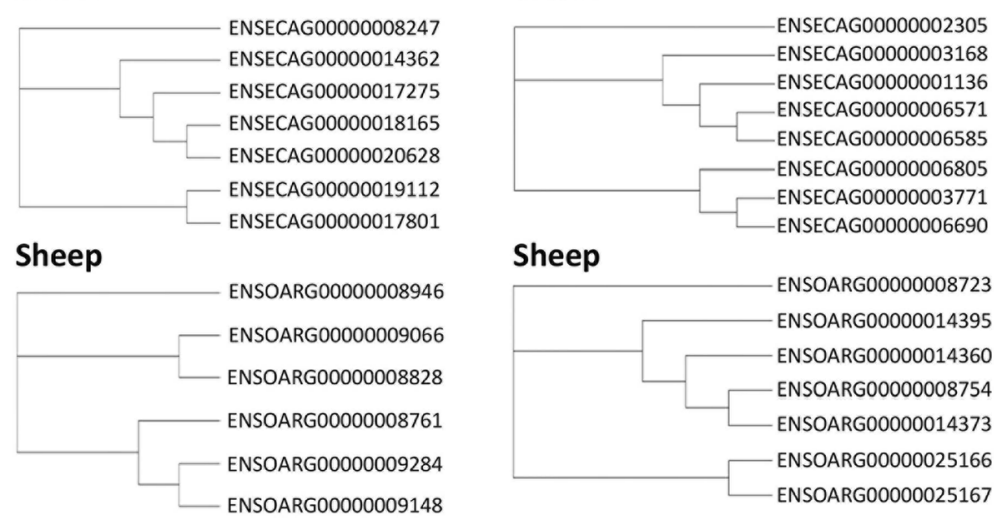

\section{Rabbit}

\section{Rabbit}
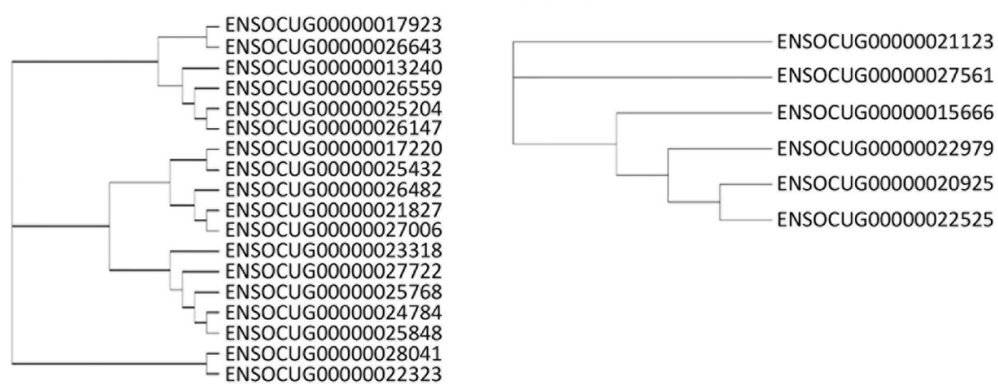

Fig. 3 Phylogenetic trees of UGT2 and IFNA gene families of 5 domestic species. Phylogenetic trees of UGT2 gene family are displayed in panel A, while the results of IFN-a gene family are showed in panel B. Only tandem cluster of genes in each family are considered. The phylogenetic trees were constructed using ClustalX (http://www.clustal.org/clustal2/) and Tree View (http://taxonomy.zoology.gla.ac.uk/rod/treeview.html)

Table 5 Enrichment fold of gene families in SD regions in 7 domestic genomes

\begin{tabular}{llllllll}
\hline & \multicolumn{2}{l}{ Species } & & & & & \\
\cline { 2 - 8 } & Cattle & Pig & Horse & Rabbit & Goat & Sheep & Chicken \\
\hline Length & 1.40 & 4.99 & 2.97 & 2.72 & 1.26 & 2.61 & 2.57 \\
Number & 1.57 & 4.07 & 2.63 & 2.03 & 1.88 & 2.38 & 1.08
\end{tabular}

To test the enrichment of gene families in SD regions, we considered the total length and the total number of genes from all gene families in each simulation, respectively. Significant enrichment was found in all 7 domestic genomes investigated $(P<0.0001)$ located in pericentromeric region of chromosome 10 and with divergence caused by inversion events. This also provided an evidence for the genomic rearrangement facilitated by segmental duplications. In addition, genes with more than 100 copies which encode spermatogenesisassociated protein were discovered in SD regions of equine genome. Prostaglandin D2 synthase $21 \mathrm{kDa}$ (brain) (PTGDS) from chicken genome had copy numbers near 100 copies, which was associated with a male-specific pathway as well [58]. Previous studies revealed that this type of multigene family consists of genes derived from 
duplication, deletion and inversion events of a common ancestral gene [55-57]. Based on our results, we suspected that segmental duplications with high identity could facilitate the occurrence of duplication, deletion and inversion events, further leading to more complex gene variation.

In the current study, 59 common genes were found in SD regions among five mammalian domestic species. These genes mainly consisted of four gene families, i.e., UGTs, IFNs, histones and ORs. UGT gene superfamily of mammalian species could be divided into four families, UGT1, UGT2, UGT3 and UGT8 [59]. All members of UGT2B family were included in these 59 common genes and the copy numbers ranged from 4-6 among different species. A previous study showed that genes in this family were closely linked among different species, but there was no evidence to prove that these genes were truly orthologous [60]. Furthermore, UGT2B17 was the most attractive one of UGT2B family and had been extensively studied previously. Polymorphic deletions were detected in UGT2B17 and UGT2B28 and segmental duplications were found near these genes [61, 62], which were associated with osteoporosis risk related to the occurrence of NAHR caused by segmental duplications [63, 64]. Thus, we suggested that the high identity and polymorphism of UGT2B gene family were strongly connected with the genomic rearrangement occurred by segmental duplications. Besides, all members of IFN alpha (IFN- $\alpha$ ) gene family were listed in the 59 common genes found in SD regions among 5 mammalian domestic species. Previous studies revealed that divergence of type I IFN was associated with rearrangements and the expansion of IFNA gene family was caused both by duplication and conversion events $[65,66]$. In the current study, common genes in the identified SD regions in multiple genomes revealed their association with immunity and response to external stimuli, especially for detoxification and drug metabolism. This might be the representative and salient characteristic of genes in SD regions. In-depth comparative analyses of function and expression of these genes among different species need to be further explored.

\section{Conclusions}

In summary, we conducted the first detailed and comparative analyses of SDs among major domestic animals to identify the SD content, characterize the feature of SDs, and annotate genes in SD regions of each species. The construction of SD maps of common domestic species offered abundant genomic resources for related studies in the future. Common genes with function of immunity and response to external stimuli were found in SD regions among the analysed mammalian domestic species. Our findings herein offer a valuable resource to facilitate both comparative genomic as well as structural genomic studies.

\section{Additional files} Additional file 1: Table S1.1-7. SD regions of 7 domestic species
detected by WSSD method. (XLSX $772 \mathrm{~kb}$ )

Additional file 2: Table S2. The distribution of SDs among 7 domestic species. (XLSX $10 \mathrm{~kb}$ )

Additional file 3: Table S3.1-7. SD regions of 7 domestic species combining results of WGAC and WSSD. (XLSX $1285 \mathrm{~kb})$

Additional file 4: Supplemental Figure. Including Figures S1-S3. (PDF $2259 \mathrm{~kb}$ )

Additional file 5: Table S4. Repeat properties of SD regions among domestic species. (XLSX 12 kb)

Additional file 6: Table S5. GO analyses of genes detected in SD regions. (XLSX 15 kb)

Additional file 7: Table S6. KEGG pathway analyses of genes detected in SD regions. (XLSX $11 \mathrm{~kb}$ )

Additional file 8: Table S7. Human homologous genes detected in SD regions. (XLSX 29 kb)

Additional file 9: Table S8. Fifty-nine human homologous genes detected in SD regions among five domestic species. (XLSX $10 \mathrm{~kb}$ )

Additional file 10: Table S9. Analyses of genes detected in SD regions based on OrthoDB. (XLSX 13 kb)

\section{Abbreviations}

CNVs: Copy number variations; GO: Gene Ontology; IFNs: Interferons; IFNa: IFN alpha; KEGG: Kyoto Encyclopedia of Genes and Genomes; LINEs: Long interspersed elements; NAHR: Non-allelic homologous recombination; NGS: Next generation sequencing; ORs: Olfactory receptors;

PTGDS: Prostaglandin D2 synthase; SDs: Segmental duplications; SINEs: Short interspersed elements; TSPY: Testis specific protein Y-encoded; UGTs: UDP glucuronosyltransferases; WGAC: Whole-genome assembly comparison; WGS: Whole genome shotgun sequencing; WSSD: Whole-genome shotgun sequence detection; ZNF: Zinc finger

\section{Acknowledgements}

We would like to thank Dr. Can Alkan at Bilkent University, Dr. Jeffrey A Bailey at University of Massachusetts Medical School and Bioinformatics Specialist John Huddleston at Howard Hughes Medical Institute for their kindly help on performing WSSD and WGAC analyses.

\section{Funding}

This work was supported by the National Major Development Program of Transgenic Breeding [2014ZX0800953B], the National Natural Science Foundations of China [31661143013], the State High-tech Development Plan [2013AA102503] and the National Natural Science Foundations of China [31272419]. Funding for open access charge: Ministry of Agriculture of China.

\section{Availability of data and materials}

All genomic data are from publicly-accessible databases and have been declared within the article. Genome assemblies for pig (Sscrofa10.2), cattle (UMD3.1), horse (EquCab2.0), rabbit (OryCun2.0), sheep (Oar_v3.1) and chicken (Gallus_gallus-4.0) are available in Ensembl databases (ftp://ftp.ensembl.org/ pub/), and those of cattle (Btau 4.6.1) and goat (CHIR_1.0) could be downloaded from the NCBI FTP site (ftp://ftp.ncbi.nlm.nih.gov/genomes/). The NGS data of porcine genome is available in DDBJ FTP site (ftp://ftp.ddbj.nig.ac.jp/ ddbj_database/dra/fastq/ERA009/ERA009086/), and ovine and caprine in NCBI FTP site (AMGL00000000.1 for sheep and AJPT00000000.1 for goat). Whole genome shotgun sequencing (WGS) sequence data of cattle, horse, rabbit and chicken are also available in NCBI FTP site (AAFC00000000.3 and DAAA00000000.2 for cattle, AAWR00000000.2 for horse, AAGW00000000.2 for rabbit and AADN00000000.3 for chicken). Related custom Perl scripts in the present study are available at https://github.com/jiang18/sd_analysis.

\section{Authors' contributions}

XTF and JCJ performed the experiments, analyzed the data, and prepared the manuscript. AP, CN, JLF, AGW and RM participated in the result interpretation and paper revision. JFL conceived and designed the 
experiments, and prepared the manuscript. All authors read and approved the final manuscript.

\section{Competing interests}

The authors declare that they have no competing interests.

\section{Consent to publication}

Not applicable.

\section{Ethics approval and consent to participate}

Ethics approval was not required in this study since genomic data were obtained from existing public datasets, and did not involve the use of animals or humans.

\section{Publisher's Note}

Springer Nature remains neutral with regard to jurisdictional claims in published maps and institutional affiliations.

\section{Author details}

${ }^{1}$ National Engineering Laboratory for Animal Breeding, Key Laboratory of Animal Genetics, Breeding and Reproduction, Ministry of Agriculture, College of Animal Science and Technology, China Agricultural University, Beijing 100193, China. ${ }^{2}$ Department of Animal and Avian Sciences, University of Maryland, College Park, MD 20740, USA. ${ }^{3}$ International Livestock Research Institute, NairobiBox 30709-00100Kenya.

Received: 19 July 2016 Accepted: 6 April 2017

Published online: 12 April 2017

\section{References}

1. Lander ES, Linton LM, Birren B, Nusbaum C, Zody MC, Baldwin J, Devon K, Dewar K, Doyle M, FitzHugh W. Initial sequencing and analysis of the human genome. Nature. 2001:409(6822):860-921.

2. Emanuel BS, Shaikh TH. Segmental duplications: an'expanding'role in genomic instability and disease. Nat Rev Genet. 2001:2(10):791-800.

3. Bailey JA, Yavor AM, Massa HF, Trask BJ, Eichler EE. Segmental duplications: organization and impact within the current human genome project assembly. Genome Res. 2001;11(6):1005-17.

4. Eichler EE. Segmental duplications: what's missing, misassigned, and misassembled_-and should we care? Genome Res. 2001;11(5):653-6.

5. Linardopoulou EV, Williams EM, Fan Y, Friedman C, Young JM, Trask BJ. Human subtelomeres are hot spots of interchromosomal recombination and segmental duplication. Nature. 2005;437(7055):94-100.

6. Perry GH, Tchinda J, McGrath SD, Zhang J, Picker SR, Cáceres AM, lafrate AJ, Tyler-Smith C, Scherer SW, Eichler EE. Hotspots for copy number variation in chimpanzees and humans. Proc Natl Acad Sci. 2006;103(21):8006-11.

7. Goidts V, Cooper DN, Armengol L, Schempp W, Conroy J, Estivill X, Nowak N, Hameister $\mathrm{H}$, Kehrer-Sawatzki $\mathrm{H}$. Complex patterns of copy number variation at sites of segmental duplications: an important category of structural variation in the human genome. Hum Genet. 2006;120(2):270-84.

8. Kim PM, Lam HY, Urban AE, Korbel JO, Affourtit J, Grubert F, Chen X, Weissman S, Snyder M, Gerstein MB. Analysis of copy number variants and segmental duplications in the human genome: Evidence for a change in the process of formation in recent evolutionary history. Genome Res. 2008; 18(12):1865-74.

9. Bailey JA, Church DM, Ventura M, Rocchi M, Eichler EE. Analysis of segmental duplications and genome assembly in the mouse. Genome Res. 2004;14(5):789-801.

10. Tuzun E, Bailey JA, Eichler EE. Recent segmental duplications in the working draft assembly of the brown Norway rat. Genome Res. 2004;14(4):493-506.

11. Cheng Z, Ventura M, She X, Khaitovich P, Graves T, Osoegawa K, Church D, DeJong P, Wilson RK, Pääbo S. A genome-wide comparison of recent chimpanzee and human segmental duplications. Nature. 2005;437(7055):88-93.

12. Nicholas TJ, Cheng Z, Ventura M, Mealey K, Eichler EE, Akey JM. The genomic architecture of segmental duplications and associated copy number variants in dogs. Genome Res. 2009:19(3):491-9.

13. Osborne LR, Li M, Pober B, Chitayat D, Bodurtha J, Mandel A, Costa T, Grebe T, Cox S, Tsui L-C. A 1.5 million-base pair inversion polymorphism in families with Williams-Beuren syndrome. Nat Genet. 2001;29(3):321-5.

14. Kuroda-Kawaguchi T, Skaletsky H, Brown LG, Minx PJ, Cordum HS, Waterston RH, Wilson RK, Silber S, Oates R, Rozen S. The AZFc region of the
Y chromosome features massive palindromes and uniform recurrent deletions in infertile men. Nat Genet. 2001;29(3):279-86.

15. Groenen MA, Archibald AL, Uenishi H, Tuggle CK, Takeuchi Y, Rothschild MF, Rogel-Gaillard C, Park C, Milan D, Megens H-J. Analyses of pig genomes provide insight into porcine demography and evolution. Nature. 2012; 491(7424):393-8.

16. Liu GE, Ventura M, Cellamare A, Chen L, Cheng Z, Zhu B, Li C, Song J, Eichler EE. Analysis of recent segmental duplications in the bovine genome. BMC Genomics. 2009:10(1):1.

17. Jiang J, Wang J, Wang H, Zhang Y, Kang H, Feng X, Wang J, Yin Z, Bao W, Zhang Q. Global copy number analyses by next generation sequencing provide insight into pig genome variation. BMC Genomics. 2014;15(1):1.

18. Bailey JA, Gu Z, Clark RA, Reinert K, Samonte RV, Schwartz S, Adams MD, Myers EW, Li PW, Eichler EE. Recent segmental duplications in the human genome. Science. 2002;297(5583):1003-7.

19. Birney E, Hudson TJ, Green ED, Gunter C, Eddy S, Rogers J, Harris JR, Ehrlich SD, Apweiler R, Austin CP. Prepublication data sharing. Nature. 2009; 461(7261):168-70

20. Zimin AV, Delcher AL, Florea L, Kelley DR, Schatz MC, Puiu D, Hanrahan F, Pertea G, Van Tassell CP, Sonstegard TS. A whole-genome assembly of the domestic cow, Bos taurus. Genome Biol. 2009:10(4):1.

21. Wade C, Giulotto E, Sigurdsson S, Zoli M, Gnerre S, Imsland F, Lear T, Adelson D, Bailey E, Bellone R. Genome sequence, comparative analysis, and population genetics of the domestic horse. Science. 2009;326(5954):865-7.

22. Gissi C, Gullberg A, Arnason U. The complete mitochondrial DNA sequence of the rabbit. Oryctolagus Cuniculus Genomics. 1998:50(2):161-9.

23. Jiang Y, Xie M, Chen W, Talbot R, Maddox JF, Faraut T, Wu C, Muzny DM, Li Y Zhang $W$. The sheep genome illuminates biology of the rumen and lipid metabolism. Science. 2014;344(6188):1168-73.

24. Hillier LW, Miller W, Birney E, Warren W, Hardison RC, Ponting CP, Bork P, Burt DW, Groenen MA, Delany ME. Sequence and comparative analysis of the chicken genome provide unique perspectives on vertebrate evolution. Nature. 2004;432(7018):695-716.

25. Elsik CG, Tellam RL, Worley KC. The genome sequence of taurine cattle: a window to ruminant biology and evolution. Science. 2009;324(5926):522-8.

26. Dong Y, Xie M, Jiang Y, Xiao N, Du X, Zhang W, Tosser-Klopp G, Wang J, Yang S, Liang J. Sequencing and automated whole-genome optical mapping of the genome of a domestic goat (Capra hircus). Nat Biotechnol. 2013;31(2):135-41.

27. Bickhart DM, Hou Y, Schroeder SG, Alkan C, Cardone MF, Matukumalli LK, Song J, Schnabel RD, Ventura M, Taylor JF. Copy number variation of individual cattle genomes using next-generation sequencing. Genome Res. 2012:22(4):778-90

28. Khatun M, Arifuzzaman M, Ashraf A. Karyotype for identification of genetic abnormalities in cattle. Asian J Anim Vet Adv. 2011:6(2):117-25.

29. Musa H, Li B, Chen G, Lanyasunya T, Xu Q, Bao W. Karyotype and Banding Patterns of Chicken Breeds. Int J Poult Sci. 2005:4(10):741-4.

30. Mota LSLS, Silva RA. Centric fusion in goats (Capra hircus): Identification of a $6 / 15$ translocation by high resolution chromosome banding. Genet Mol Biol. 1998;21(1). https://dx.doi.org/10.1590/S1415-47571998000100012.

31. Richer C, Power M, Klunder L, McFeely R, Kent M. Standard karyotype of the domestic horse (Equus caballus). Hereditas. 1990;112(3):289-93.

32. SCHRÖDER J, LOO W. Comparison of karyotypes in three species of rabbit: Oryctolagus cuniculus, Sylvilagus nuttallii and S. idahoensis. Hereditas. 1979; 91(1):27-30.

33. Hansen K. Identification of the chromosomes of the domestic pig (Sus scrofa domestica). An identification key and a landmark system. Genet Sel Evol. 1977;9(4):1.

34. HANSEN-MELANDER E, MELANDER Y. The karyotype of the pig. Hereditas. 1974;77(1):149-58

35. Takagi N, Sasaki M. A phylogenetic study of bird karyotypes. Chromosoma. 1974:46(1):91-120.

36. Hansen K. The karyotype of the domestic sheep (Ovis aries) identified by quinacrine mustard staining and fluorescence microscopy. Hereditas. 1973; 75(2):233-40.

37. Alkan C, Kidd JM, Marques-Bonet T, Aksay G, Antonacci F, Hormozdiari F, Kitzman JO, Baker C, Malig M, Mutlu O. Personalized copy number and segmental duplication maps using next-generation sequencing. Nat Genet. 2009:41(10):1061-7.

38. Ayers KL, Davidson NM, Demiyah D, Roeszler KN, Grützner F, Sinclair AH, Oshlack A, Smith CA. RNA sequencing reveals sexually dimorphic gene 
expression before gonadal differentiation in chicken and allows comprehensive annotation of the W-chromosome. Genome Biol. 2013;14(3):1.

39. Zhao Q, Zhu Z, Kasahara M, Morishita S, Zhang Z. Segmental duplications in the silkworm genome. BMC Genomics. 2013;14(1):1.

40. Chan F, Cianfriglia M, Echard G, Fox R, Gustavsson I, Martin DP, Nesbitt M. Standard karyotype of the laboratory rabbit, Oryctolagus cuniculus. Cytogenet Cell Genet. 1981;31(4):240-8.

41. Strassburg CP, Nguyen N, Manns MP, Tukey RH. UDPglucuronosyltransferase activity in human liver and colon. Gastroenterology. 1999;116(1):149-60.

42. Kondrashov FA. Gene duplication as a mechanism of genomic adaptation to a changing environment. Proc R Soc Lond B Biol Sci. 2012;279(1749):5048-57.

43. Niimura $Y$, Nei M. Extensive gains and losses of olfactory receptor genes in mammalian evolution. PLoS One. 2007;2(8):e708.

44. Kondrashov FA, Rogozin IB, Wolf YI, Koonin EV. Selection in the evolution of gene duplications. Genome Biol. 2002;3(2):1.

45. Ando Y, Saka H, Ando M, Sawa T, Muro K, Ueoka H, Yokoyama A, Saitoh S, Shimokata K, Hasegawa Y. Polymorphisms of UDP-glucuronosyltransferase gene and irinotecan toxicity: a pharmacogenetic analysis. Cancer Res. 2000; 60(24):6921-6.

46. Fensterl V, Sen GC. Interferons and viral infections. Biofactors. 2009;35(1):14-20.

47. Ohta T. Evolution of gene families. Gene. 2000;259(1-2):45-52.

48. Kriventseva EV, Tegenfeldt F, Petty TJ, Waterhouse RM, Simão FA, Pozdnyakov IA, loannidis $P$, Zdobnov EM. OrthoDB v8: update of the hierarchical catalog of orthologs and the underlying free software. Nucleic Acids Res. 2015:43(D1):D250-6.

49. Cheung J, Estivill X, Khaja R, MacDonald JR, Lau K, Tsui LC, Scherer SW. Genome-wide detection of segmental duplications and potential assembly errors in the human genome sequence. Genome Biol. 2003;4(4):R25.

50. Kelley DR, Salzberg SL. Detection and correction of false segmental duplications caused by genome mis-assembly. Genome Biol. 2010;11(3):R28.

51. Burt DW. Chicken genome: current status and future opportunities. Genome Res. 2005;15(12):1692-8.

52. Berlin S, Ellegren $\mathrm{H}$. Chicken W: a genetically uniform chromosome in a highly variable genome. Proc Natl Acad Sci U S A. 2004;101(45):15967-9.

53. Hamilton C, Favetta L, Di Meo G, Floriot S, Perucatti A, Peippo J, Kantanen J, Eggen A, lannuzzi L, King W. Copy number variation of testis-specific protein, Y-encoded (TSPY) in 14 different breeds of cattle (Bos taurus). Sex Dev. 2009:3(4):205-13.

54. Schnieders F, Dörk T, Arnemann J, Vogel T, Werner M, Schmidtke J. Testisspecific protein, Y-encoded (TSPY) expression in testicular tissues. Hum Mol Genet. 1996;5(11):1801-7.

55. Tang M, Waterman M, Yooseph S. Zinc finger gene clusters and tandem gene duplication. J Comput Biol. 2002;9(2):429-46.

56. Tunnacliffe A, Liu L, Moore JK, Leversha MA, Jackson MS, Papi L, FergusonSmith MA, Thiesen $\mathrm{H}-J$, Ponder A. Duplicated KOX zinc finger gene clusters flank the centromere of human chromosome 10: evidence for a pericentric inversion during primate evolution. Nucleic Acids Res. 1993;21(6):1409-17.

57. Savard OT, Bertrand D, El-Mabrouk N. Evolution of orthologous tandemly arrayed gene clusters. BMC Bioinf. 2011;12(9):1.

58. Moniot B, Boizet-Bonhoure B, Poulat F. Male specific expression of lipocalintype prostaglandin D synthase (CPTGDS) during chicken gonadal differentiation: relationship with cSOX9. Sex Dev. 2008;2(2):96-103.

59. Mackenzie PI, Bock KW, Burchell B, Guillemette C, Ikushiro S-i, lyanagi T, Miners JO, Owens IS, Nebert DW. Nomenclature update for the mammalian UDP glycosyltransferase (UGT) gene superfamily. Pharmacogenet Genomics. 2005;15(10):677-85.

60. Tukey RH, Strassburg CP. Genetic multiplicity of the human UDPglucuronosyltransferases and regulation in the gastrointestinal tract. Mol Pharmacol. 2001;59(3):405-14.

61. Guillemette C, Lévesque E, Harvey M, Bellemare J, Menard V. UGT genomic diversity: beyond gene duplication. Drug Metab Rev. 2010;42(1):24-44

62. Turgeon D, Carrier J-S, Lévesque É, Beatty BG, Bélanger A, Hum DW. Isolation and characterization of the human UGT2B15 gene, localized within a cluster of UGT2B genes and pseudogenes on chromosome 4. J Mol Biol. 2000;295(3):489-504

63. Ménard V, Eap O, Harvey M, Guillemette C, Lévesque É. Copy-number variations (CNVs) of the human sex steroid metabolizing genes UGT2B17 and UGT2B28 and their associations with a UGT2B15 functional polymorphism. Hum Mutat. 2009;30(9):1310-9.
64. Yang T-L, Chen X-D, Guo Y, Lei S-F, Wang J-T, Zhou Q, Pan F, Chen Y, Zhang Z-X, Dong S-S. Genome-wide copy-number-variation study identified a susceptibility gene, UGT2B17, for osteoporosis. Am J Hum Genet. 2008; 83(6):663-74.

65. Walker AM, Roberts RM. Characterization of the bovine type I IFN locus: rearrangements, expansions, and novel subfamilies. BMC Genomics. 2009;10(1):1.

66. Woelk CH, Frost SD, Richman DD, Higley PE, Pond SLK. Evolution of the interferon alpha gene family in eutherian mammals. Gene. 2007;397(1):38-50

\section{Submit your next manuscript to BioMed Central and we will help you at every step:}

- We accept pre-submission inquiries

- Our selector tool helps you to find the most relevant journal

- We provide round the clock customer support

- Convenient online submission

- Thorough peer review

- Inclusion in PubMed and all major indexing services

- Maximum visibility for your research

Submit your manuscript at www.biomedcentral.com/submit
) Biomed Central 